\title{
Alternative Assets and Cryptocurrencies
}

\author{
Christian M. Hafner
}

Louvain Institute of Data Analysis and Modeling, Université catholique de Louvain, 1348 Louvain-la-Neuve, Belgium; christian.hafner@uclouvain.be

Received: 19 December 2019; Accepted: 20 December 2019; Published: 3 January 2020

\begin{abstract}
Alternative assets, defined by their low correlation with classical financial assets, have become an important investment vehicle in times of negative interest rates and in the aftermath of the global economic and financial crisis. Hedge funds increasingly invest in physical assets such as fine art, wine, or diamonds. Although digital and not physical, cryptocurrencies share many features of alternative assets, but are hampered by high volatility, sluggish commercial acceptance, and regulatory uncertainties. This special issue covers a broad variety of topics in financial technology, and provides a state-of-the-art overview of cryptocurrencies from economic, financial, statistical and technical points of view.
\end{abstract}

Keywords: fintech; alternative investments; digital finance; blockchain; bitcoin

This special issue collects high profile research papers on the innovative topic of alternative assets and cryptocurrencies. It aims at providing a guideline and inspiration for both researchers and practitioners in financial technology. Alternative assets such as fine art, wine or diamonds have become popular investment vehicles in the aftermath of the global financial crisis. Triggered by low correlation with classical financial markets, diversification benefits arise for portfolio allocation and risk management. Cryptocurrencies share many features of alternative assets, but are hampered by high volatility, sluggish commercial acceptance, and regulatory uncertainties. Moreover, they are particularly prone to the development of speculative bubbles, as in the recent price surge of 2017, which has been documented and confirmed by statistical tests, see e.g., Cheah and Fry (2015); Cheung et al. (2015); Corbet et al. (2017); Hafner (2018) and Su et al. (2018).

Nonetheless, the new area of digital finance increasingly attracts academic and general interest. The appearance of journal special issues, even of new journals such as Digital Finance from Springer, dedicated entirely to this new field, and the creation of cryptocurrency indices such as the CRIX (see Trimborn and Härdle 2018), are but a few examples. General review articles are Böhme et al. (2015) for bitcoin, and Härdle et al. (2018) for cryptocurrencies in general.

The papers comprised in this special issue address alternative assets and cryptocurrencies from economic, financial, statistical, and technical points of view. It gives an overview of the current state of the art and helps to understand their properties and prospects using innovative approaches and methodologies. The timeliness of this collection is apparent from the view and download statistics of the journal's website, where at the time of this writing, most of the papers are in the top ten over the last year or more, which highlights the general interest in the topic.

A first challenge is the analysis of time series properties such as volatility, including financial applications. Conrad et al. (2018) study long and short term volatility components and find that Bitcoin volatility is closely linked to indicators of global economic activity. Henriques and Sadorsky (2018) use multivariate GARCH-type models to show that there is an economic value for risk averse investors to replace gold by Bitcoin in investment portfolios. Kjærland et al. (2018) identify dynamic pricing factors for Bitcoin using autoregressive distributed lags (ADL) and GARCH. They find that the Google search indicator and returns on the S\&P 500 stock index are significant pricing factors. 
A second block of papers deals with high frequency data for cryptocurrencies, meaning minute-stamped or transaction data. A common theme is predictability, which is confirmed in several papers, and which would violate classical concepts of market efficiency. Fischer et al. (2019) use a specific trading strategy to show that there are statistical arbitrage opportunities in the cross-section of cryptocurrencies. In a deep learning framework, Shintate and Pichl (2019) propose a so-called random sampling method for trend prediction classification, applied to high frequency Bitcoin prices. Catania and Sandholdt (2019) find predictability at high frequencies up to six hours, but not at higher aggregation levels, while realized volatility is characterized by long memory and leverage effects. Schnaubelt et al. (2019) study the properties of Bitcoin limit order books. Their findings suggest that, while many features are similar to classical financial markets, the distributions of trade sizes, and limit order prices are rather distinct, and liquidity costs are relatively high.

Third, a few papers deal with peculiarities of cryptocurrencies such as initial coin offerings, proof-of-work protocols and sentiment indices. Ante et al. (2018) investigate blockchain-based initial coin offerings (ICOs) and find that they exhibit similarities to classical crowdfunding and venture capital markets, including the determinants of success factors. Bocart (2018) proposes a new proof-of-work protocol to establish consensus about transactions to be added to the blockchain, arguing that the availability of alternatives to the classical SHA256 algorithm used by Bitcoin reduces the risk of attacks against particular proof-of-work protocols. Finally, Chen and Hafner (2019) use a publicly available crypto-market sentiment index as an explanatory variable for locally explosive behavior of crypto prices and volatility. In a smooth transition autoregressive model, they identify bubble periods for Bitcoin and the CRIX, a crypto market index.

Last, but not least, we indeed have a paper that deals with a "classical" alternative asset, that is, diamonds. Jotanovic and D'Ecclesia (2019) show that, perhaps counterintuitively, investing in diamond mining stocks is not a valid alternative to investing in diamond commodity directly. Moreover, diamond stock returns are not driven by diamond price dynamics, but rather by local market stock indices.

All of the above papers cover many diverse aspects of alternative assets and cryptocurrencies that we hope will contribute to the already rich literature and become useful resources and inspirations for anyone working in the exciting new field of financial technology.

Funding: This research received no external funding.

Conflicts of Interest: The author declares no conflict of interest.

\section{References}

Ante, Lennart, Philipp Sandner, and Ingo Fiedler. 2018. Blockchain-Based ICOs: Pure Hype or the Dawn of a New Era of Startup Financing? Journal of Risk and Financial Management 11: 80. [CrossRef]

Bocart, Fabian. 2018. Inflation Propensity of Collatz Orbits: A New Proof-of-Work for Blockchain Applications. Journal of Risk and Financial Management 11: 83. [CrossRef]

Böhme, Rainer, Nicolas Christin, Benjamin Edelman, and Tyler Moore. 2015. Bitcoin: Economics, Technology, and Governance. Journal of Economic Perspectives 29: 213-38. [CrossRef]

Catania, Leopoldo, and Mads Sandholdt. 2019. Bitcoin at High Frequency. Journal of Risk and Financial Management 12: 36. [CrossRef]

Cheah, Eng-Tuck, and John Fry. 2015. Speculative bubbles in Bitcoin markets? An empirical investigation into the fundamental value of Bitcoin. Economics Letters 130: 32-36. [CrossRef]

Chen, Cathy H., and Christian M. Hafner. 2019. Sentiment-Induced Bubbles in the Cryptocurrency Market. Journal of Risk and Financial Management 12: 53. [CrossRef]

Cheung, Adrian (Wai-Kong), Eduardo Roca, and Jen-Je Su. 2015. Crypto-currency bubbles: an application of the Phillips-Shi-Yu (2013) methodology on Mt. Gox bitcoin prices. Applied Economics 47: 2348-58. [CrossRef]

Conrad, Christian, Anessa Custovic, and Eric Ghysels. 2018. Long- and Short-Term Cryptocurrency Volatility Components: A GARCH-MIDAS Analysis. Journal of Risk and Financial Management 11: 23. [CrossRef] 
Corbet, Shaen, Brian Lucey, and Larisa Yarovya. 2017. Datestamping the Bitcoin and Ethereum Bubbles. Finance Research Letters 26: 81-88. [CrossRef]

Fischer, Thomas G., Christopher Krauss, and Alexander Deinert. 2019. Statistical Arbitrage in Cryptocurrency Markets. Journal of Risk and Financial Management 12: 31. [CrossRef]

Hafner, Christian M. 2018. Testing for Bubbles in Cryptocurrencies with Time-Varying Volatility. Journal of Financial Econometrics. [CrossRef]

Härdle, Wolfgang K., Campbell R. Harvey, and Raphael CG Reule. 2018. Understanding Cryptocurrencies. IRTG Discussion Paper. Berlin: Humboldt University.

Henriques, Irene, and Perry Sadorsky. 2018. Can Bitcoin Replace Gold in an Investment Portfolio? Journal of Risk and Financial Management 11: 48. [CrossRef]

Jotanovic, Vera, and Rita L. D'Ecclesia. 2019. Do Diamond Stocks Shine Brighter than Diamonds? Journal of Risk and Financial Management 12: 79. [CrossRef]

Kjærland, Frode, Aras Khazal, Erlend Krogstad, Frans Nordstrøm, and Are Oust. 2018. An Analysis of Bitcoin's Price Dynamics. Journal of Risk and Financial Management 11: 63. [CrossRef]

Schnaubelt, Matthias, Jonas Rende, and Christopher Krauss. 2019. Testing Stylized Facts of Bitcoin Limit Order Books. Journal of Risk and Financial Management 12: 25. [CrossRef]

Shintate, Takuya, and Lukáš Pichl. 2019. Trend Prediction Classification for High Frequency Bitcoin Time Series with Deep Learning. Journal of Risk and Financial Management 12: 17. [CrossRef]

$\mathrm{Su}$, Chi-Wei, Zheng-Zheng Li, Ran Tao, and Deng-Kui Si. 2018. Testing for multiple bubbles in bitcoin markets: A generalized sup ADF test. Japan and the World Economy 46: 56-63. [CrossRef]

Trimborn, Simon, and Wolfgang Karl Härdle. 2018. CRIX an Index for cryptocurrencies. Journal of Empirical Finance 49: 107-22. [CrossRef]

(C) 2020 by the author. Licensee MDPI, Basel, Switzerland. This article is an open access article distributed under the terms and conditions of the Creative Commons Attribution (CC BY) license (http://creativecommons.org/licenses/by/4.0/). 